\title{
Are Topical Skin Adhesives an Option for Wound Closure Following Musculoskeletal Oncology Surgery? A STROCSS-compliant Observational Study
}

Thanapon Chobpenthai ( $\nabla$ thanapon.cho@pccms.ac.th )

HRH Princess Chulabhorn College of Medical Science https://orcid.org/0000-0002-2482-8203

Thanate Poosiripinyo

Khon Kaen University Faculty of Medicine

Pakjai Tuntarattanapong

Prince of Songkla University Faculty of Medicine

Pichaya Thanindratarn

Chulabhorn Hospital

Warayos Trathitephun

Chulabhorn Hospital

Research article

Keywords: 2-octyl cyanoacrylate, topical skin adhesive, musculoskeletal oncology surgery

Posted Date: January 11th, 2021

DOl: https://doi.org/10.21203/rs.3.rs-142776/v1

License: (c) (1) This work is licensed under a Creative Commons Attribution 4.0 International License.

Read Full License 


\section{Abstract}

Background:

2-octyl cyanoacrylate (20CA) is a high-viscosity medical-grade tissue adhesive used routinely. However, no studies have evaluated its use in musculoskeletal oncology surgery.

Methods:

We enrolled 99 patients undergoing musculoskeletal oncology surgery. 20CA was chosen for wound closure, and it was applied by a specific surgeon for all patients. Drying times for the adhesive were recorded, and photographs were obtained intra-operatively. Post-treatment follow-up constituted queries regarding pain level, and recording incisional dehiscence, wound infection, hematoma, and incisional bleeding. Data collection was performed post-operatively at 48 hours, 5-10 days, 14 days, and 30 days. Other adverse events were documented.

Results:

20CA was applied to 110 incisions in 99 patients constituting 62 female patients and 37 male patients. The mean age of patients was $50.41( \pm 16.83)$ years; mean incision length was $10.24( \pm 5.7) \mathrm{cm}$; and the mean pain score using a visual analogue scale (VAS) was 2.37 on post-operative day 7 . The mean drying time was $1.81( \pm 0.59)$ minutes; $91(91 \%)$ patients reported excellent and superior satisfaction, and the remaining patients reported "good" (6\%) and "fair" (2\%) satisfaction. The percentage of dehiscence, hematoma, and keloid formation was considerably low.

Conclusion:

20CA was safe in musculoskeletal oncology surgical incisions in this study. The incidence of postoperative adverse events was low. However, some patients developed a hematoma. Post-operative pain was low, and patient satisfaction was high. 20CA can be a practical alternative to traditional suture closure for skin incisions after musculoskeletal surgery.

\section{Introduction}

Surgery in oncology patients demands additional measures and precautions owing to the patient's health condition and possible repeat surgical interventions. Intra- and post-operatively, wound healing remains a major concern, occasionally owing to patients' vulnerability to infection. The body's wound healing process serves as a major line of defense against infection, and cancer may impact the natural healing efficiency by impairing the cellular plasticity of the epidermal cell populations [1]. Cascading and orchestrated signaling pathways that are involved in normal wound healing are distorted with cancer development [2]. The roles of stem cell plasticity [3] and stroma [4] are altered during cancer development and metastasis, which significantly affects wound healing. Macrophages, which are part of the innate 
wound healing response, are used by cancer cells in disease metastasis [5]; hence, focusing on rapid wound healing intra- or post-operatively is important.

In modern surgery, topical skin adhesives (TSA) are being chosen over traditional sutures for better and rapid wound healing. Octyl-2-cyanoacrylate or 2-octyl-cyanoacrylate (2OCA) skin adhesives are preferred compared with sutures because of the painless process and their rapid action. Comparisons of sutures and adhesives have been performed for facial wound healing [6], wound closure in cholecystectomy incisions [7], congenital cleft lip [8], and in other surgical processes [9]. These studies reported better outcomes with TSA compared with sutures.

20CA skin adhesive has been found to be effective and superior in many applications. A comparative analysis suggested that adhesives are effective and better than staples in managing wounds related to incisional surgical site complications (SSCs) [10]. Similarly, 20CA was found to be effective compared with sutures in another study [11]. Clinically relevant characteristics have also been evaluated for different skin adhesives that are presently being used routinely, and these adhesives were found suitable [12]. Moreover, in vivo evaluation helped establish the superiority of octyl-cyanoacrylate-based adhesives compared with adhesives that are butyl-cyanoacrylate-based [13]. Hence, in oncology-related surgery, where wound healing could be an issue, TSAs may provide an alternative support to achieve better and rapid wound healing. However, certain aspects, such as allergic reactions, and other possible adverse events should be addressed before careful application of any topical skin adhesive.

Musculoskeletal oncology requires a complex surgical process, often demanding repeat operations, depending on the disease condition. Hence, using TSA, especially 20CA, could be a better choice. Skin adhesive-based treatment can be considered because it involves less skin wedging compared with traditional sutures, especially with recurrent tumors requiring re-operation. The benefits of using this approach includes 1) fast skin closure, which is particularly helpful in oncology patients as it is wellknown that the more the operative time, the more is the risk of infection; 2) no skin mark along the wound as observed in traditional sutures. In case of tumor recurrence, it is not required to wedge the skin over the sutures; hence, there is less skin loss; 3 ) easy wound care as no dressing is required and it is water resistant; 4) good cosmetic outcomes; and 5) painless wound healing.

In addition, no recent studies have reported about TSA use in musculoskeletal surgery. Thus, this present study aimed to report that the application of 2OCA is safe and effective for musculoskeletal oncology surgical wound healing. The primary objective of this retrospective study was understanding the clinical outcomes and possible adverse events following the application of 20CA in topical incision closure for musculoskeletal surgery. As secondary outcomes, we evaluated the drying time and patients' satisfaction with the wound scar.

\section{Materials And Methods}

\section{Study design}


Topical skin adhesive is a modern complementary addition to traditional sutures, and the application of 20CA has been encouraging in many aspects in this context. The present retrospective study was complying with STROCSS guideline [14] and designed to understand the effectiveness of 2OCA skin adhesive application for wound closure after musculoskeletal surgery in cancer patients. The study was conducted at Chulabhorn Cancer Center, Bangkok, Thailand, from 08/28/2017 to 08/17/2020.

\section{Study participants and eligibility criteria}

A total of 99 participants were selected for the present study following strict eligibility criteria. Both sexes were included in the study. The inclusion and exclusion criteria for the participants were as follows:

Inclusion criteria: (1) Diagnosis with soft tissue or bone tumor (including metastasis), tumor-like lesion in extremities and back area; (2) Any age and sex; (3) Use 20CA for skin closure

Exclusion criteria: (1) Not clear diagnosis; (2) Previous surgical wound before surgery; (3) Allergy to 20CA; (4) Pre-operative radiotherapy; (5) Skin disorder

\section{Surgical specifications and patient characteristics}

We carefully recorded the incision characteristics, such as the specific upper and lower extremities and back region, and the diagnosis requiring surgery, such as malignant soft tissue tumors, benign soft tissue tumors, malignant bone tumors, benign bone tumors, and bone metastasis. All incisions were linear vertical incisions.

\section{Post-operative treatment and follow-up}

The focus of the study was surgical closure of the skin wound in adult patients using 20CA, which was applied by a specifically designated surgeon. We recorded adhesive drying times and performed photography intra-operatively. Post-treatment follow-up was conducted with queries of patients' pain levels assessed using a visual analogue scale (VAS), as well as evaluation of incision dehiscence, wound infection, hematoma, and incisional bleeding. Follow-up was performed post-operatively at 48 hours, 510 days, 14 days, and 30 days. Specific intra-operative regimens were followed where wound edges were manually approximated by fingers or forceps. The physicians painted the 20CA over the manually apposed wound edges with the applicator tip and were careful not to apply adhesive between the wound edges. The wound was held for drying. For each incision, the drying times were tested by a light touch of the forceps to ensure that the wound was closed, and the drying time was recorded. We closed deep layer and subcutaneous layer with Vicryl $1 / 0$ and $2 / 0$ or $3 / 0$, respectively, before applying the topical skin adhesive in all cases.

\section{Ethical considerations}

This study was approved by the Human Research Ethics Committee of our institution (Chulabhorn Cancer Center, Bangkok, Thailand; project code: 054/2563), and the study was performed in accordance with the 
ethical standards of the 1975 Declaration of Helsinki.

\section{Data collection and statistical analysis}

A total of 15 parameters, diagnosis, demographic, surgery-related, wound healing, side effects, infection, and patient outcome-related parameters, were considered in the present study. All statistical analyses were conducted using $\mathrm{R}$ (version 3.6.1). A descriptive analysis of each parameter was performed. Numerical data are presented as mean \pm standard deviation, and ordinal and categorical data are presented as percentages. The numerical variables were further analyzed for quartile distributions and are represented as box plots. Histograms and bar plots were considered, as appropriate. Correlation analysis was conducted using Pearson's r, Spearman's rho, and Kendall's Tau-b method to understand the possible relationship between the wound and other parameters. The significance of sex-based differences was evaluated using Pearson's Chi-square test.

\section{Results}

In accordance with the study objective, each parameter was inspected carefully, and statistical assessment was performed for descriptive and inferential observations. The specific objective of these observations was to understand the possible relationships between the important parameters, such as sex difference, age, wound length, and drying time. However, every variable was keenly inspected for any plausible impacts they may have had on patient outcomes.

\section{Clinical observations}

All resections involved linear vertical incisions. Some patients developed hematoma after almost 2 weeks of surgery, and examples are presented in Figure 1 and Figure 2 as observed post-operatively.

\section{Observations and outcomes}

Most of the subjects who participated in this study were aged between 40 and 70 years (Fig. 3A). The mean patients' age was $50.41( \pm 16.83$ ) years (Fig. 3B). The mean age of the female patients was 50.46 $( \pm 16.36)$ years, and the mean age of the male patients was $50.32( \pm 17.80)$ years. The total number of female participants in this study was $62(62.62 \%)$, and the number of male participants was 37 (37.37\%) of the 99 subjects selected for the study (Fig. 3C).

All selected participants underwent proper diagnosis and were categorized on the basis of the diagnostic (Dx) categories (Table 1) and the type of cancer (Fig. 3D). The Dx categories constituted 26 cases of lipoma (26.26\%) and 14 cases of liposarcoma (14.14\%), followed by ganglion cyst $(n=8,8.08 \%)$ (Table 1). Metastasis was observed in $13 / 99$ cases $(13.13 \%)$.

Patients' diagnoses were categorized on the basis of the type of the tumor, benign, malignant, or metastatic. Additionally, in the benign group, benign bone tumors $(n=10,10 \%)$ and benign soft tissue tumor $(n=47,47.47 \%)$ were diagnosed and recorded. Similarly, in the case of malignancy, malignant 
bone tumor $(n=3,3.03 \%)$ and malignant soft tissue tumors $(n=26,26.26 \%)$ were identified. Bone metastasis was observed in 13 cases (Fig. 3D).

Inspection and demographics of the tumor or diagnosed cancer were also performed on the basis of the body part of origin (Fig. 3E) to understand the most predominant body part affected by musculoskeletal cancer; 59 cases (59.59\%) had musculoskeletal cancer in the upper extremities and back, and, in contrast, $40(40.40 \%)$ cases had cancer in the lower extremities. Most cancers in the upper extremities were observed in the shoulder, forearm, and hand (Fig. 3E); several cases were recorded in the back region as well. The thigh and hip were the most affected areas in the lower body (Fig. 3E).

Analysis of the pain intensity due to the surgical wounds and the scars was performed using a VAS (Fig. $3 F$ ), with a scale range of $1-4$, and with most patients reporting scores of 2 or 3 (Fig. 3F). Hematoma was observed in 10 patients during the post-operative follow-up (Fig. 3G). Table 1 presents the distribution of the diagnosed cases on the basis of the Dx category and shows a predominance of lipoma $(n=26,26 \%)$, liposarcoma $(n=14,14 \%)$, and metastasis $(n=13,13 \%)$ (Table 1$)$.

We further analyzed the surgical operations that were performed, which constituted the following: curettage and cementation and intramedullary nailing (IMN); marginal resection; curettage, cementation and cephalomedullary nailing (CMN); curettage, cementation and IMN; curettage, cementation, and plating; endoprosthesis; hemiarthroplasty; marginal resection; open biopsy; and wide resection. The majority of the surgeries were performed either with marginal resection $(n=44,44 \%)$ or by wide resection $(n=40,40 \%)$ (Table 2).

The distribution of the wound length, drying time, and patients' responses are shown in Figure 4. Most surgical wounds were within $15 \mathrm{~cm}$ (Fig. 4A), and the mean incision length was $10.24( \pm 5.7) \mathrm{cm}$ (Fig. 4B).

The mean drying time was $1.81( \pm 0.59)$ minutes. In most cases, the adhesive dried within 2 minutes after application (Fig. 4C). All patients were requested to provide feedback on the treatment outcomes (Fig. 4D). Of the 99 patients, 91 (91\%) patients reported excellent satisfaction with the treatment approach (Fig. 4D). The remainder of the patients reported scores of "good" (6\%) and "fair" (2\%). No negative feedback was received from any of the patients regarding the use of the adhesive as a method of surgical wound treatment and closure.

\section{Adverse event assessment}

Careful monitoring was performed for possible adverse events intra- and post-operatively. Two patients developed minor infections, keloid formation was observed in five patients, and poor wound healing as dehiscence was observed in $7 \%$ of patients. Post-operative incisional bleeding was recorded in $3 \%$ of the patients, and hematoma was observed in 10 patients (Fig. 3G).

\section{Statistical evaluation of wound length and drying time}


We performed a correlation analysis for the wound length and drying time (Fig. 5), with separate analyses for the male (Fig. 5A) and female (Fig. 5B) patients. Although the correlation values were similar between the sexes (Fig. $5 \mathrm{C}-\mathrm{E}$ ), the relationship between the wound length and drying time was significant for both male patients $(P=0.019)$ and female patients $(P=0.010)$.

These findings motivated us to conduct Pearson's Chi-square test to detect statistically significant observations that differed between the male and female patients. However, for the considered parameters, no statistically significant difference was observed for the observed outcomes between the sexes (Table 3).

\section{Discussion}

Sutures have been used traditionally for wound closure and recovery; however, they have mechanical and physical effects and psychological impact on the patient that alter the healing process. Modern wound closure techniques include staples and adhesives as well as sutures [15]. However, there are discrepancies regarding selection of the closure technique intra-operatively [16-17]. Several studies attempted to compare these techniques to understand the advantages and disadvantages related to specific contexts, such as arthroplasty [18-19].

Different procedures and techniques have been adopted as wound closures after musculoskeletal surgery in cancer patients, micro-surgery [20] and vacuum-assisted wound closure for complicated wounds affected by radiation and following resection of musculoskeletal tumors [21-22]. However, complications associated with post-operative wound healing, especially for musculoskeletal oncology patients, remain a challenge. Reports have been available for such complications in soft tissue-associated sarcoma for decades [23-25]. Several associated factors, such as dehiscence, infection, and fistula, have been examined to understand their effects in complicated surgical wounds [26].

Developing applications and growing interest in using TSAs have led to better patient outcomes as reported in a previous study of the prevalence of TSA application in oncology patients [27]. 20CA skin adhesives are being chosen more often for their better post-operative healing outcomes in oncology patients. Specific applications have also been reported in single-incision laparoscopic surgery in colorectal cancer patients [28]. In addition, the comparative economic and clinical outcomes associated with 20CA as an effective skin adhesive have been studied very recently [29-30].

The availability of reports evaluating 20CA in musculoskeletal cancer patient treatment is limited. The present study was conducted to understand the clinical outcomes, patient satisfaction regarding pain relief, and adverse events following the application of 20CA skin adhesive for post-operative wound healing in musculoskeletal oncology patients. The observations and outcomes were satisfactory, with most patients highly satisfied with the results of the adhesive in wound healing. However, a small number of patients developed hematoma and incisional bleeding. 
Keloid formation was observed in 5 patients. Three of these 5 patients also had wound dehiscence. So, wound dehiscence may be one factor for developing keloid formation in our series. True incidence and prevalence of keloid is not known as no population study has assessed the epidemiology of this disorder. In his 2001 publication, Marneros [31] stated that "reported incidence of keloids in the general population ranges from a high of $16 \%$ among the adults in Zaire to a low of $0.09 \%$ in England," quoting from Bloom's 1956 publication on heredity of keloids [32]. Clinical observations show that the disorder is more common among sub-Saharan Africans, African Americans and Asians, with unreliable and very wide estimated prevalence rates ranging from $4.5-16 \%[33,34]$.

A significant statistical relationship between wound length and drying time was observed for both men and women. Hence, using TSA in minimal surgical incisions accelerates recovery and reduces the scar. These observations suggest that TSA can be a better alternative to sutures for surgical wound healing even in musculoskeletal oncology patients with long incisions. TSA is a painless remedy, as shown in the VAS scores in the present study. However, other important factors, such as allergic reactions [35], wound length, hematoma, and keloid should be carefully evaluated before TSA application.

The present study indicated that most cases required surgery in their upper extremities compared with the lower extremities. The average wound length in the upper and lower extremities and the back region was $8.53( \pm 4.75) \mathrm{cm}, 12.25( \pm 6.69) \mathrm{cm}$, and $10.25( \pm 3.27) \mathrm{cm}$, respectively. Similarly, the average respective drying times for the upper and lower extremities, and the back were $1.63( \pm 0.52)$ mins, $1.92( \pm 0.65)$ mins, and $2.16( \pm 0.38)$ mins, respectively. An abundance of female patients was observed in this study compared with male patients, and marginal and wide resection was chosen, depending on the assessment of the disease conditions. Statistically, a significant relationship was observed between drying time and wound length for both sexes.

\section{Study Limitation}

The present study was a single-center study with a small sample size. Multi-center studies with larger and more diverse samples may provide conclusive and generalized insight to support the observations recorded in the present study. In addition, a large randomized controlled trial may provide interesting insight for applying skin adhesives and their better impact on wound recovery.

\section{Conclusion}

Our study outcomes suggested that the application of 20CA is safe and effective for surgical wound healing. The number of adverse events, such as incisional bleeding, hematoma, keloid, and dehiscence was low. Rapid drying time also supported shorter operation time and quick post-operative recovery. Hence, skin adhesives can be considered effective, painless wound healing treatments for musculoskeletal oncology patients as well as for recurrent sarcoma.

\section{List Of Abbreviations}


20CA, 2-octyl cyanoacrylate

VAS, visual analogue scale

TSA, topical skin adhesives

Dx, diagnostic

IMN, intramedullary nailing

$\mathrm{CMN}$, cephalomedullary nailing

\section{Declarations}

\section{Competing interests}

The authors declare that they have no competing interests.

\section{Funding}

All authors certify that no affiliations with or involvement in any organization or entity with any financial interest, or non-financial interest.

\section{Consent for publication}

All Patients acknowledged and signed informed consent regarding publishing their data and photographs.

\section{Ethics approval and consent to participate}

The Human Research Ethics Committee of the Chulabhorn Research Institute acknowledged and approved this study. (Approval Number: RAA 054/2563). Informed consent was deemed not applicable since the study is purely retrospective in nature, and therefore written informed consent for participation was not obtained for adults or children under 16 years of age.

\section{Availability of data and materials}

The datasets used and/or analysed during the current study are available from the corresponding author on reasonable request.

\section{Authors's contributions}

TC Conceptualization, Methodology, Validation, Formal analysis, Investigation, Data Curation, Writing Original Draft, Visualization, Supervision, Project administration. TP Validation, Formal analysis, Investigation, Writing - Original Draft. PAT Methodology, Validation, Formal analysis, Writing - Review \& 
Editing. PIT Investigation, Data Curation, Visualization, Writing - Review \& Editing. WT Methodology, Investigation, Data Curation, Writing - Review \& Editing.

\section{Acknowledgement}

We thank Jane Charbonneau, DVM, from Edanz Group (https://en-author-services.edanzgroup.com/ac) for English editing a draft of this manuscript.

\section{References}

1. Rognoni E, Watt FM. Skin cell heterogeneity in development, wound healing, and cancer. Trends Cell Biol 2018;28:709-22.

2. Sundaram GM, Quah S, Sampath P. Cancer: the dark side of wound healing. FEBS J 2018;285:451634.

3. Ge Y, Fuchs E. Stretching the limits: from homeostasis to stem cell plasticity in wound healing and cancer. Nat Rev Genet 2018;19:311.

4. Huet E, Jaroz C, Nguyen HQ, et al. Stroma in normal and cancer wound healing. FEBS J 2019;286:2909-20.

5. Muliaditan T, Caron J, Okesola M, et al. Macrophages are exploited from an innate wound healing response to facilitate cancer metastasis. Nat Commun 2018;9:1-15.

6. Handschel JGK, Depprich RA, Dirksen D, et al. A prospective comparison of octyl-2-cyanoacrylate and suture in standardized facial wounds. Int J Oral Maxillofac Surg 2006;35:318-23.

7. Jallali N, Haji A, Watson CJ. A prospective randomized trial comparing 2-octyl cyanoacrylate to conventional suturing in closure of laparoscopic cholecystectomy incisions. J Laparoendosc Adv Surg Tech A 2004;14:209-11.

8. Knott PD, Zins JE, Banbury J, et al. A comparison of dermabond tissue adhesive and sutures in the primary repair of the congenital cleft lip. Ann Plast Surg 2007;58:121-5.

9. Pronio A, Di Filippo A, Narilli P, et al. Closure of cutaneous incision after thyroid surgery: a comparison between metal clips and cutaneous octyl-2-cyanoacrylate adhesive. A prospective randomized clinical trial. Eur J Plast Surg 2011;34:103-10.

10. Flick KF, Simpson RE, Soufi M, et al. Comparison of skin closure techniques in patients undergoing open pancreaticoduodenectomy: A single center experience. Am J Surg 2020;220:972-5.

11. Chavan S, Dnyanmote A, Sinha N. Comparative study of the effectiveness of the octyl-2cyanoacrylate as a tissue adhesive for skin closure \& skin closure by non absorbable sutures (nylon). J Med Sci Clin Res 2016;4:13179-81.

12. Singer AJ, Perry L. A comparative study of the surgically relevant mechanical characteristics of the topical skin adhesives. Acad Emerg Med 2012;19:1281-6.

13. Singer AJ, Perry LC, Allen Jr RL. In vivo study of wound bursting strength and compliance of topical skin adhesives. Acad Emerg Med 2008;15:1290-4. 
14. Agha R, Abdall-Razak A, Crossley E, Dowlut N, losifidis C, Mathew G; STROCSS Group. STROCSS 2019 Guideline: Strengthening the reporting of cohort studies in surgery. Int J Surg. 2019 Dec;72:156165.

15. Waheed A, Council M. Wound closure techniques. Treasure Island (FL): StatPearls Publishing; 2017.

16. Armitage J, Lockwood S. Skin incisions and wound closure. Surgery (Oxf) 2011;29:496-501.

17. Hochberg J, Meyer KM, Marion MD. Suture choice and other methods of skin closure. Surg Clin North Am 2009;89:627-41.

18. Eggers MD, Fang L, Lionberger DR. A comparison of wound closure techniques for total knee arthroplasty. J Arthroplasty 2011;26:1251-8.

19. Opanova M, Hasegawa I, Unebasami E, et al. Comparison of wound complications between two closing techniques following total hip arthroplasty via the direct anterior approach. J Hip Surg 2019;3:191-6.

20. Kontogeorgakos VA, Eward WC, Brigman BE. Microsurgery in musculoskeletal oncology. Eur J Orthop Surg Traumatol 2019;29:271-8.

21. Siegel HJ, Long JL, Watson KM, Fiveash JB. Vacuum-assisted closure for radiation-associated wound complications. J Surg Oncol 2007;96:575-82.

22. Bickels J, Kollender Y, Wittig JC, et al. Vacuum-assisted wound closure after resection of musculoskeletal tumors. Clin Orthop Relat Res 2005;441:346-50.

23. Peat BG, Bell RS, Davis $A$, et al. Wound-healing complications after soft-tissue sarcoma surgery. Plastic Reconstr Surg 1994;93:980-7.

24. Geller DS, Hornicek FJ, Mankin HJ, Raskin KA. Soft tissue sarcoma resection volume associated with wound-healing complications. Clin Orthop Relat Res 2007;459:182-5.

25. Abouarab MH, Salem IL, Degheidy MM, et al. Therapeutic options and postoperative wound complications after extremity soft tissue sarcoma resection and postoperative external beam radiotherapy. Int Wound J 2018;15:148-58.

26. González CVS, de Carvalho VF, Kim SHP, et al. Complicated surgical wounds and associated factors in oncology patients. Plastic Surg Nurs 2020;40:91-9.

27. Zhao H, He Y, Huang $\mathrm{H}$, et al. Prevalence of medical adhesive-related skin injury at peripherally inserted central catheter insertion site in oncology patients. J Vasc Access 2018;19:23-7.

28. Hirano Y, Hiranuma C, Douden K, Hattori M. Wound closing method with 2-octyl cyanoacrylate after single-incision laparoscopic surgery for colorectal cancer. Eur Surg 2019;51:27-30.

29. Sutton N, Schmitz N-D, Johnston SS. Economic and clinical comparison of 2-octyl cyanoacrylate/polymer mesh tape with skin staples in total knee replacement. J Wound Care 2018;27:S12-22.

30. Johnston SS, Fortin SP, Pracyk JB, et al. Economic and clinical outcomes of spinal fusion surgeries with skin closure through skin staples plus waterproof wound dressings versus 2-octyl cyanoacrylate plus polymer mesh tape. Spine J 2020; Sep 3:S1529-9430(20)31075-5. 
31. Marneros AG, Norris JEC, Olsen BR, Reichenberger, E. Clinical Genetics of Familial Keloids. Arch Dermatol 2001;137(11):1429-34.

32. Bloom D. Heredity of keloids; review of the literature and report of a family with multiple keloids in five generations. NY State J Med 1956;56(4):511-9.

33. Froelich K, Staudenmaier R, Kleinsasser N, Hagen R. Therapy of auricular keloids: review of different treatment modalities and proposal for a therapeutic algorithm. Eur Arch Otorhinolaryngol 2007;264(12):1497-508.

34. Gauglitz G, Korting H, Pavicic T, Ruzicka T, Jeschke MG. Hypertrophic scarring and keloids: Pathomechanisms and current and emerging treatment strategies. Mol Med 2011;17(1-2):113-25.

35. Davis MD, Stuart MJ. Severe allergic contact dermatitis to Dermabond Prineo, a topical skin adhesive of 2-octyl cyanoacrylate increasingly used in surgeries to close wounds. Dermatitis 2016;27:75-6.

\section{Tables}


Table 1

Observed distribution of the diagnosis codes among the participants.

\begin{tabular}{|lll|}
\hline Dx Code & Frequency & Percent \\
\hline Desmoid fibromatosis & 2 & 2.020 \\
\hline Epidermal inclusion cyst & 1 & 1.010 \\
\hline Ewing's sarcoma & 2 & 2.020 \\
\hline Fibrosis & 2 & 2.020 \\
\hline GCT of the distal radius & 6 & 6.061 \\
\hline GCT of bone & 2 & 2.020 \\
\hline GCT of the tendon sheath & 5 & 5.051 \\
\hline Ganglion cyst & 8 & 8.081 \\
\hline Hemangioma & 3 & 3.030 \\
\hline Leiomyosarcoma & 1 & 1.010 \\
\hline Lipoma & 26 & 26.263 \\
\hline Liposarcoma & 14 & 14.141 \\
\hline MPNST & 1 & 1.010 \\
\hline Metastasis & 13 & 13.13 \\
\hline Osteosarcoma & 1 & 1.010 \\
\hline PNST & 3 & 3.030 \\
\hline Sarcomatoid sarcoma & 1 & 1.010 \\
\hline UPS & 3 & 3.030 \\
\hline Fibrosarcoma & 1 & 1.010 \\
\hline Hemangioma & 1 & 1.010 \\
\hline Leiomyosarcoma & 3 & 3.030 \\
\hline Total & 99 & 100.000 \\
\hline
\end{tabular}

GCT, giant cell tumor; MPNST, malignant peripheral nerve sheath tumor, PNST, peripheral nerve sheath tumor; UPS, undifferentiated pleomorphic sarcoma 
Table 2

Patient categorization according to the surgery.

\begin{tabular}{|lll|}
\hline Surgery type & Frequency & Percent \\
\hline Curettage and cementation and IMN & 1 & 1.010 \\
\hline Marginal resection & 5 & 5.051 \\
\hline Curettage and cementation and CMN & 3 & 3.030 \\
\hline Curettage and cementation and IMN & 1 & 1.010 \\
\hline Curettage and cementation and plating & 1 & 1.010 \\
\hline Endoprosthesis & 2 & 2.020 \\
\hline Hemiarthroplasty & 1 & 1.010 \\
\hline Marginal resection & 44 & 44.444 \\
\hline Open biopsy & 1 & 1.010 \\
\hline Wide resection & 40 & 40.404 \\
\hline Total & 99 & 100.000 \\
\hline
\end{tabular}

IMN, intramedullary nailing; $\mathrm{CMN}$, cephalomedullary nailing

Table 3

Evaluation of statistically significant differences between the sexes using Pearson's Chi-square test.

\begin{tabular}{|llll|}
\hline Parameter & Chi-squared & Degree of freedom & p-value \\
\hline Age & 747.71 & 756 & 0.578 \\
\hline Dx & 128.58 & 156 & 0.9469 \\
\hline Diagnosis & 9.8284 & 12 & 0.631 \\
\hline Operation & 28.386 & 28 & 0.4441 \\
\hline Body Part & 54.48 & 63 & 0.7692 \\
\hline Wound Length & 122.68 & 156 & 0.9773 \\
\hline VAS & 5.5561 & 9 & 0.7834 \\
\hline Drying time & 1.031 & 4 & 0.9051 \\
\hline Patient satisfaction & 0.25628 & 2 & 0.8797 \\
\hline
\end{tabular}

Dx, diagnostic classification; VAS, visual analogue scale 


\section{Figures}
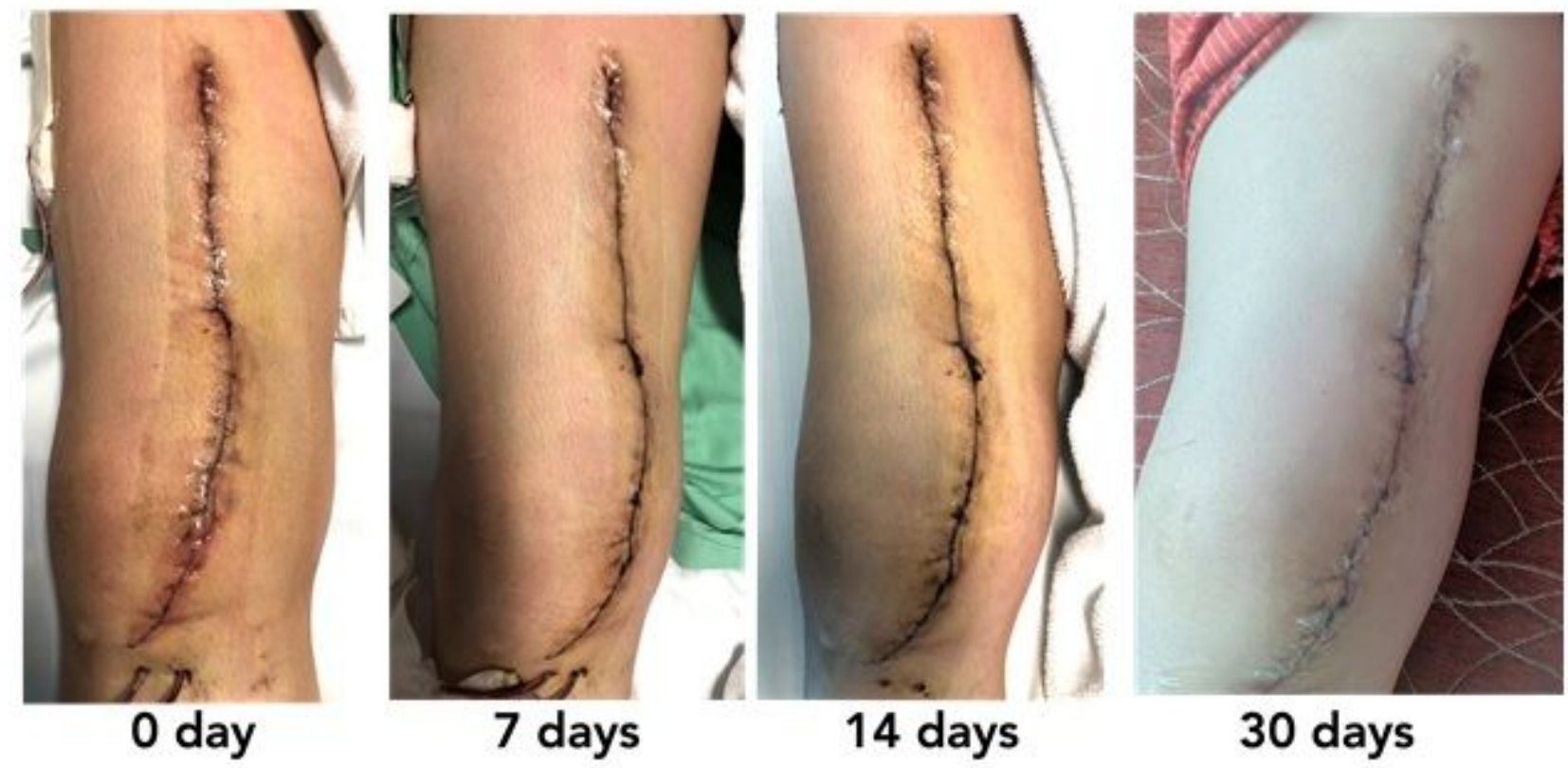

Figure 1

Representative case of wide resection of the distal femur and reconstruction with an endoprosthesis. The outcome indicated appropriate wound healing with no complications, and excellent patient satisfaction was recorded. 


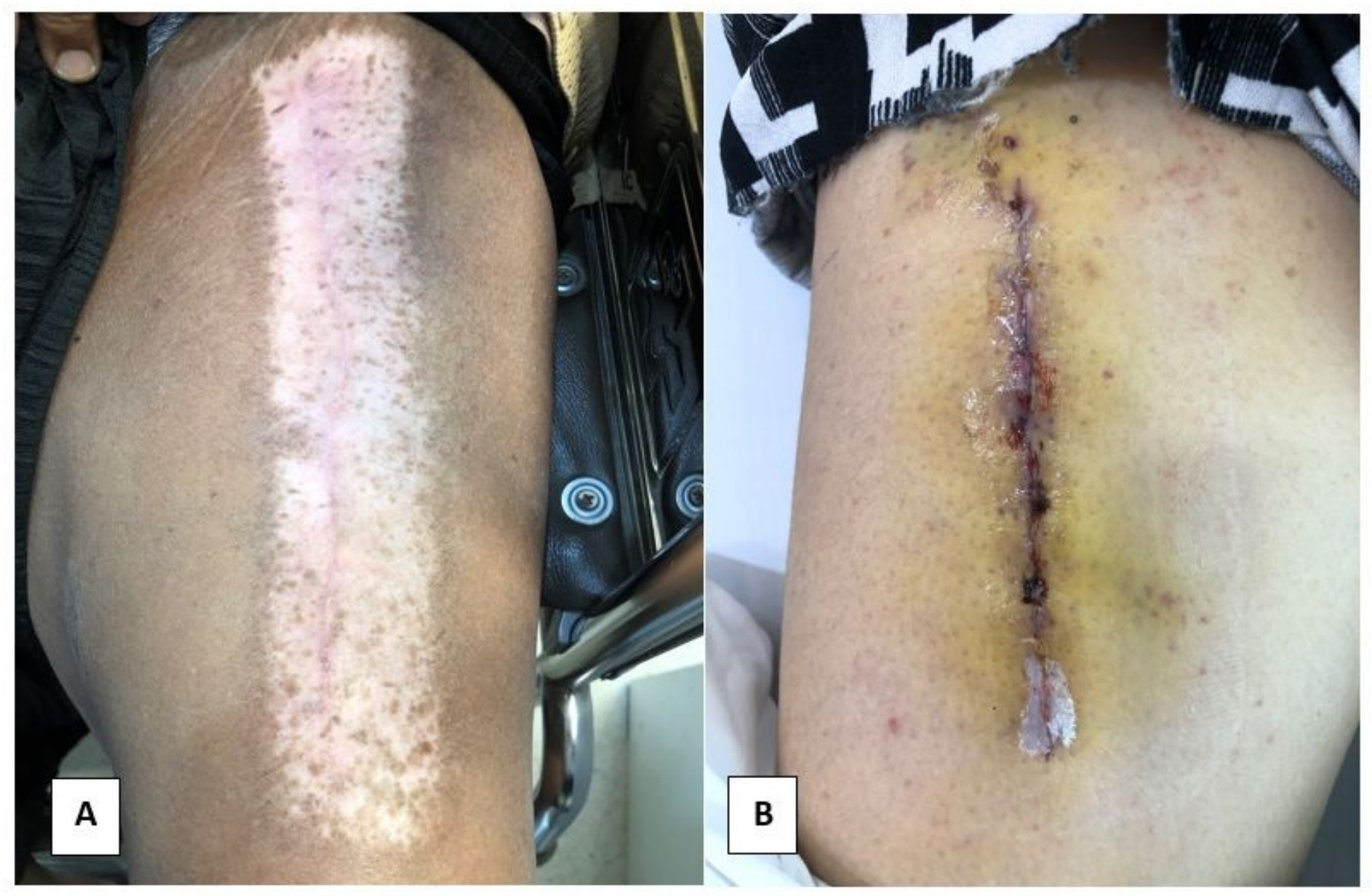

Figure 2

(A) Post-operative findings 180 days after wide resection and post-operative radiotherapy (RT) 60 days after liposarcoma resection in the left thigh. (B) Example of a post-operative hematoma in a patient's right thigh on day 14 . 

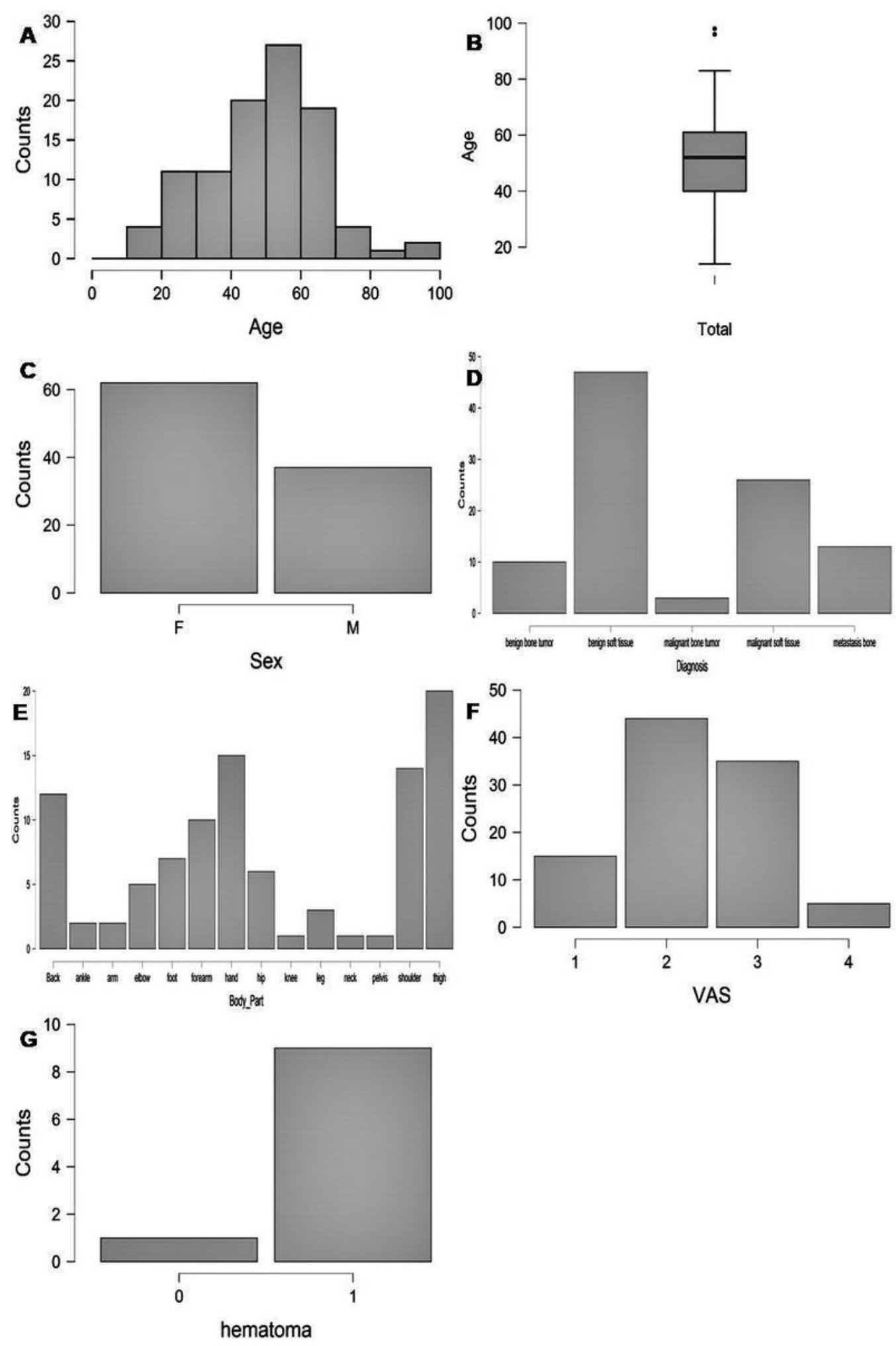

\section{Figure 3}

Distribution of the descriptive parameters. (A) Patients' age distribution; (B) Box plot presenting the 25th, 50th, and 75th percentile of the age distribution; (C) Distribution of the patients on the basis of sex; (D) Diagnosis-related outcome distribution; (E) Body part/region undergoing operation and wound treatment; (F) Wound healing outcomes according to the visual analogue scale (VAS) scores, and; (G) Frequency of post-operative hematoma. 

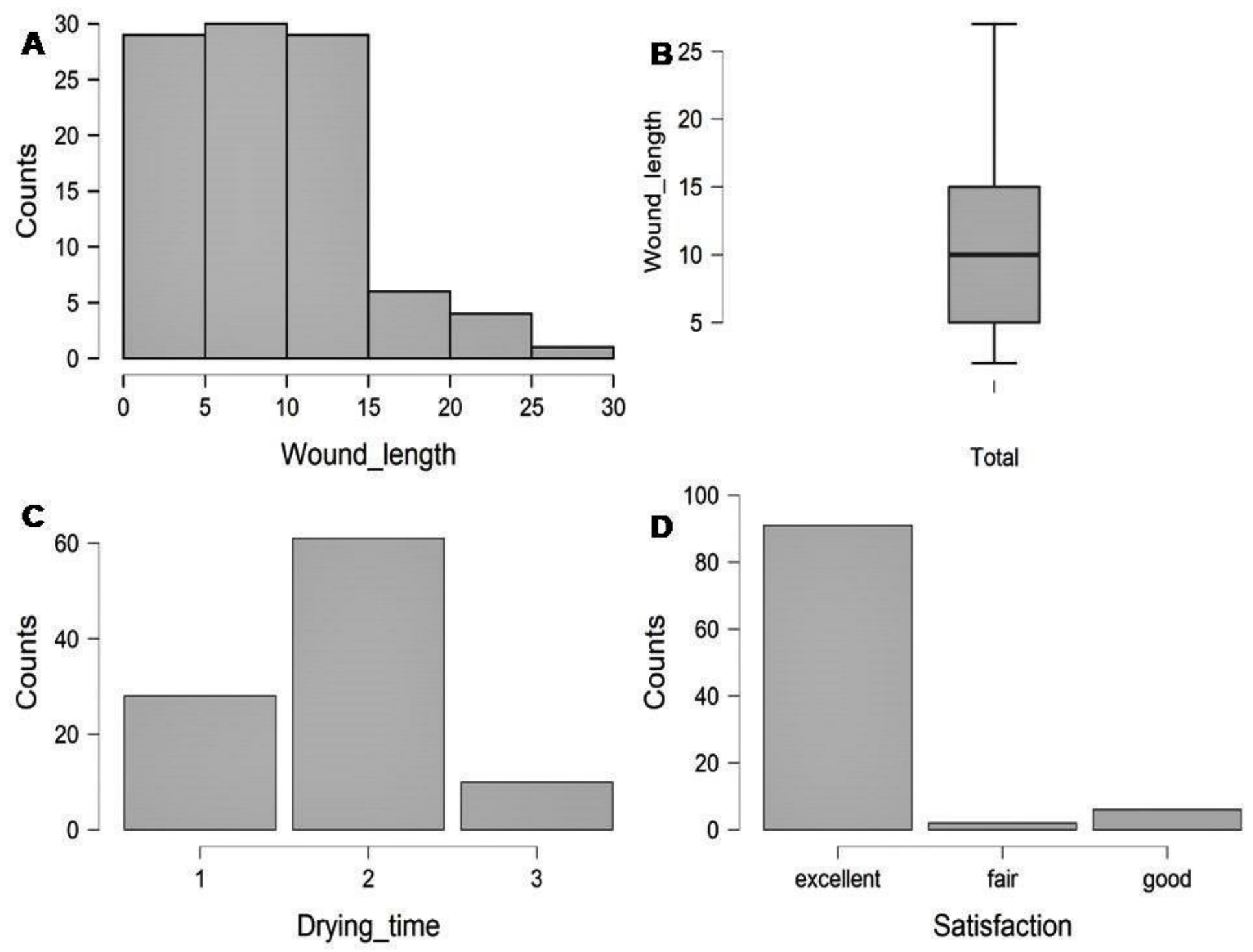

Figure 4

Observations related to (A) wound length; (B) box plot of wound length; (C) wound drying time, and; (D) patient satisfaction survey results. 

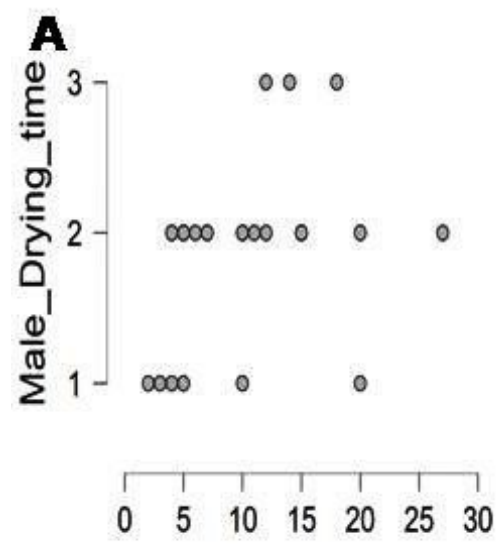

Male_Wound_length

$$
\begin{aligned}
r & =0.310 \\
95 \% \mathrm{Cl}:[-0.015,0.576] & \\
\rho & =0.383 \\
95 \% \mathrm{Cl}: & {[0.067,0.629] } \\
\tau & =0.330
\end{aligned}
$$

$95 \% \mathrm{Cl}:[0.138,0.521]$
$B$
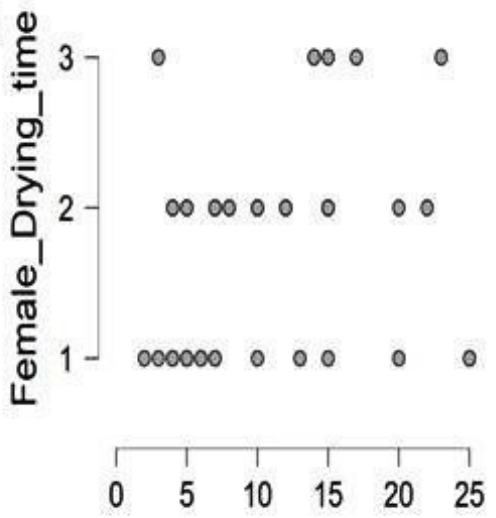

Female_Wound_length

\section{Pearson's r}

D Spearman's tho

\section{E Kendall's tau B}

$0.259^{*} 0.1440 .276$ Female_Wound_length -
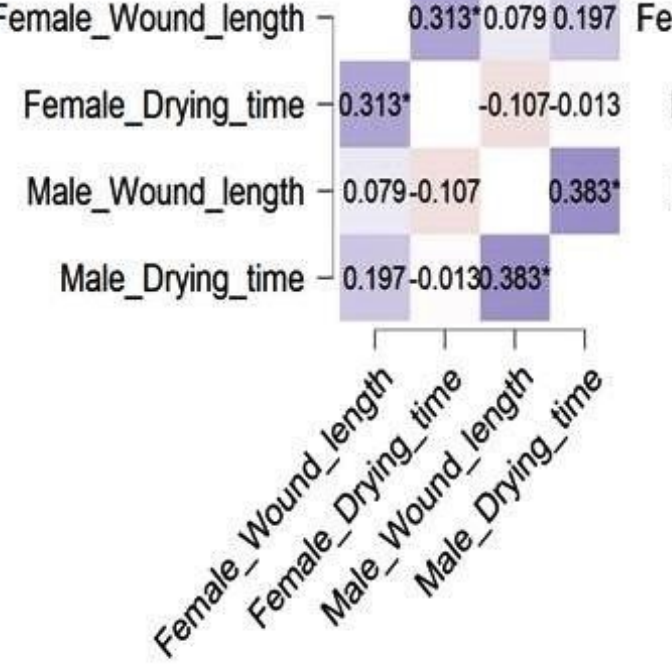

$r=0.259$

$95 \% \mathrm{Cl}:[0.010,0.478]$

$$
\rho=0.313
$$

$95 \% \mathrm{Cl}:[0.069,0.522]$

$\tau=0.273$

$95 \% \mathrm{Cl}:[0.104,0.443]$
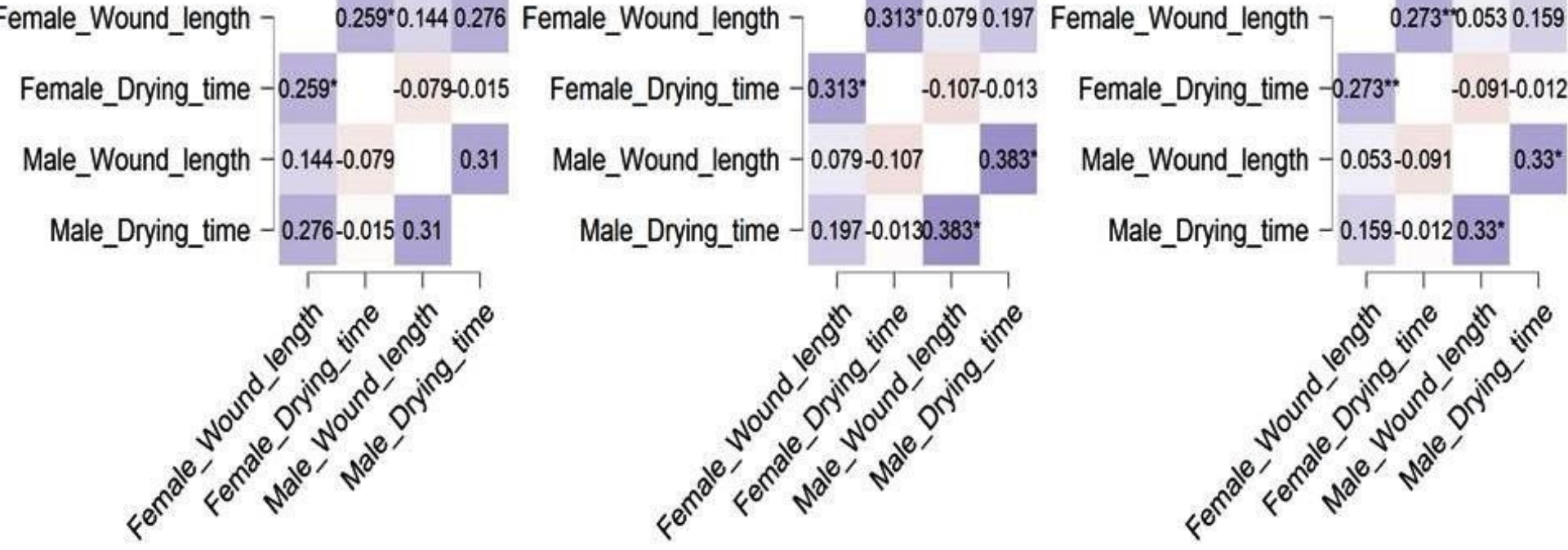

\section{Figure 5}

Comparative sex-based statistical correlation analysis results of wound length and drying time. (A) Wound length and drying time in male subjects and (B) wound length and drying time in female subjects. Statistical correlation and heatmap representation using (C) Pearson's r correlation, (D) Spearman's rho correlation, and (E) Kendall's Tau-b correlation for wound length and drying time for both sexes.

\section{Supplementary Files}

This is a list of supplementary files associated with this preprint. Click to download.

- strocss2019checklist.docx 